\title{
Twin epidemics: the effects of HIV and systolic blood pressure on mortality risk in rural South Africa, 2010-2019
}

Brian Houle ${ }^{1,2^{*}}$, Chodziwadziwa W Kabudula ${ }^{2}$, Andrea M Tilstra ${ }^{3,4,5}$, Sanyu A Mojola ${ }^{2,6}$, Enid Schatz ${ }^{2,7}$, Samuel J Clark ${ }^{2,8}$, Nicole Angotti ${ }^{2,9}$, F Xavier Gómez-Olivé ${ }^{2}$ and Jane Menken ${ }^{4}$

\begin{abstract}
Background: Sub-Saharan African settings are experiencing dual epidemics of HIV and hypertension. We investigate effects of each condition on mortality and examine whether HIV and hypertension interact in determining mortality.

Methods: Data come from the $2010 \mathrm{Ha}$ Nakekela population-based survey of individuals ages 40 and older $(1,802$ women; 1,107 men) nested in the Agincourt Health and socio-Demographic Surveillance System in rural South Africa, which provides mortality follow-up from population surveillance until mid-2019. Using discrete-time event history models stratified by sex, we assessed differential mortality risks according to baseline measures of HIV infection, HIV-1 RNA viral load, and systolic blood pressure.
\end{abstract}

Results: During the 8-year follow-up period, mortality was high (477 deaths). Survey weighted estimates are that $37 \%$ of men (mortality rate $987.53 / 100,000,95 \%$ Cl: 986.26 to 988.79 ) and $25 \%$ of women (mortality rate 937.28/100,000, 95\% Cl: 899.7 to 974.88) died. Over a quarter of participants were living with HIV (PLWH) at baseline, over $50 \%$ of whom had unsuppressed viral loads. The share of the population with a systolic blood pressure of $140 \mathrm{~mm} \mathrm{Hg}$ or higher increased from $24 \%$ at ages $40-59$ to $50 \%$ at ages 75 -plus and was generally higher for those not living with HIV compared to PLWH. Men and women with unsuppressed viral load had elevated mortality risks (men: adjusted odds ratio (aOR) 3.23, 95\% Cl: 2.21 to 4.71, women: aOR 2.05, 95\% Cl: 1.27 to 3.30). There was a weak, non-linear relationship between systolic blood pressure and higher mortality risk. We found no significant interaction between systolic blood pressure and HIV status for either men or women ( $p>0.05)$.

Conclusions: Our results indicate that HIV and elevated blood pressure are acting as separate, non-interacting epidemics affecting high proportions of the older adult population.

PLWH with unsuppressed viral load were at higher mortality risk compared to those uninfected. Systolic blood pressure was a mortality risk factor independent of HIV status. As antiretroviral therapy becomes more widespread, further longitudinal follow-up is needed to understand how the dynamics of increased longevity and multimorbidity among people living with both HIV and high blood pressure, as well as the emergence of COVID-19, may alter these patterns.

Keywords: Mortality, Rural, South Africa, HIV, Hypertension, Blood pressure

*Correspondence: brian.houle@anu.edu.au

${ }^{1}$ School of Demography, The Australian National University, Canberra, Australia

Full list of author information is available at the end of the article

\section{Background}

South Africa is undergoing complex demographic and epidemiological transitions, with intersecting epidemics of HIV and hypertension. The country has one of the largest and fastest growing older populations in 
sub-Saharan Africa [1]. Globally, South Africa has one of the highest levels of HIV prevalence and the largest antiretroviral therapy (ART) program [2]. The success of widespread ART rollout has led to population-level declines in mortality $[3,4]$, resulting in the aging of the epidemic as people living with HIV (PLWH) who are on ART live longer [5]. However, gaps remain in the HIV treatment cascade - amongst adults aged 40-plus in rural South Africa, $63 \%$ of those screened HIV-positive were on ART and, of those, $72 \%$ had viral suppression [6]. The burden of noncommunicable diseases (NCD) is also high [7], particularly the prevalence of hypertension $[8,9]$. This is complicated by low awareness among those with a hypertensive condition: an estimated $38-64 \%$ of hypertensive people were aware of their status and only $8-23 \%$ had controlled blood pressure [9-11].

With widespread availability of ART in sub-Saharan Africa and resulting survival gains, it is critical to understand the evolving morbidity and mortality patterns of PLWH. Studies in high income countries have shown high levels of cardiovascular risk factors and events in PLWH [12-14], and mixed evidence on shifts in causes of death from AIDS to NCDs $[15,16]$. Limited evidence from sub-Saharan Africa indicates persistent HIV-related hospitalizations and mortality due to AIDS despite being on ART [17-20].

It is unclear if HIV infection and ART interact with hypertension to increase mortality risk. Studies in Kenya and Haiti restricted to PLWH found associations between blood pressure and elevated mortality [21, 22]. However, a U.S. study of veterans living with HIV matched to uninfected veterans in care found that HIV and blood pressure were independently associated with mortality [23].

We use a population-based cross-sectional survey (ages 40-plus) and eight-year longitudinal mortality follow-up data from rural South Africa to examine associations between HIV status and systolic blood pressure and differential mortality risk.

\section{Methods}

\section{Setting and data}

Our data come from the 2010-2011 Ha Nakekela HIV and NCD Study and the Agincourt Health and socioDemographic Surveillance System (Agincourt HDSS), situated in the rural northeast of South Africa. The Agincourt HDSS has collected longitudinal, populationlevel data annually on all vital events and socio-economic information since 1992 [24]. In 2011, the surveillance population comprised approximately 90,000 people residing in 27 villages. For the Ha Nakekela study, we randomly selected a sex-age stratified sample of 7,662 individuals using the 2009 Agincourt HDSS census round as the sampling frame, including an oversample of older adults $[25,26]$. Inclusion criteria were men and women aged 15 and older who were permanent residents in the Agincourt HDSS study area based on information from the 2009 census round. A total of 4,362 individuals were eligible, consented to be interviewed and tested for HIV [26]. Of these, 3,024 were aged 40-plus. Data collected included blood tests and dried blood spots, anthropometric and blood pressure measurements, and a sexual behavior and chronic disease risk factor questionnaire. Follow-up information on mortality came from annual census update rounds of the Agincourt HDSS through mid-2019.

\section{Key measures}

HIV status [25]

Dried blood spots were tested using screening assay Vironostika Uniform 11 (Biomeriuex, France). Positive results were retested using the SD Bioline HIV ELISA test (SD; Standard Diagnostics Inc., Korea). If the two tests were inconsistent, a third assay was conducted (Elecys, Roche, USA) to determine the final result.

\section{Blood pressure [8]}

Blood pressure was measured three times using a Boso BP instrument [27-29]. We used the average of the second and third measurements. Current use of antihypertensive medications was based on self-report.

\section{Analyses}

We restricted this analysis to the 3,024 respondents aged 40-plus and, further, to those with complete covariate information, resulting in a final analytic sample of 2,909 adults: 1,802 women and 1,107 men. We restricted to those aged 40-plus as the risk of dying from systolic blood pressure-related diseases at younger ages was too low for systematic analysis. Baseline covariates included age, nationality (South African or other), marital status (single, married/cohabiting, widowed/divorced), years of education (0-3 years, 4-8 years, 8+ years), employment status $(1 / 0)$, and household wealth tertile based on a household asset index [30]).

The primary covariates were HIV and systolic blood pressure. We categorized HIV as a combination of HIV diagnosis and viral load: (1) HIV negative; (2) HIV positive, viral load suppressed; and (3) HIV positive, viral load unsuppressed. Respondents were classified as suppressed if their viral load was less than 400 copies $/ \mathrm{mL}$ and unsuppressed if their viral load was above 400 copies/mL [31]. In supplementary analyses, we assessed the sensitivity of our analysis to this threshold by considering a second HIV status variable that classified respondents as suppressed if their viral load was less than 1,000 copies/mL and unsuppressed if their viral load was at least 
Table 1 Descriptive statistics and tests of differences between men and women for the estimation sample, Agincourt, South Africa, 2010-11

\begin{tabular}{|c|c|c|c|c|}
\hline & $\begin{array}{l}\text { Overall } \\
\text { Percent (\%) }\end{array}$ & $\begin{array}{l}\text { Men } \\
\text { Percent (\%) }\end{array}$ & $\begin{array}{l}\text { Women } \\
\text { Percent (\%) }\end{array}$ & $\begin{array}{l}P \text {-value } \\
\text { Difference }\end{array}$ \\
\hline Age Group & & & & 0.068 \\
\hline 40-59 & 51.3 & 48.8 & 52.5 & \\
\hline $60-74$ & 24.7 & 27.8 & 23.3 & \\
\hline $75+$ & 24.0 & 23.4 & 24.3 & \\
\hline Missing (N) & 0 & 0 & 0 & \\
\hline HIV Status & & & & 0.003 \\
\hline HIV Negative & 73.0 & 73.0 & 73.0 & \\
\hline HIV Positive Suppressed ${ }^{\mathrm{a}}$ & 11.5 & 8.6 & 12.8 & \\
\hline HIV Positive, Unsuppressed ${ }^{b}$ & 15.5 & 18.4 & 14.2 & \\
\hline Missing $(\mathrm{N})$ & 226 & 117 & 109 & \\
\hline Citizenship & & & & 0.007 \\
\hline South African & 71.6 & 75.3 & 69.8 & \\
\hline Other & 28.4 & 24.7 & 30.2 & \\
\hline Missing (N) & 0 & 0 & 0 & \\
\hline Marital Status & & & & $<0.001$ \\
\hline Single & 15.1 & 18.1 & 13.7 & \\
\hline Married/cohabiting & 46.7 & 60.1 & 40.3 & \\
\hline Widowed/divorced & 38.2 & 21.7 & 46.1 & \\
\hline Missing (N) & 3 & 1 & 2 & \\
\hline Education Level & & & & $<0.001$ \\
\hline None/very low ( $\leq 3$ yrs) & 46.8 & 40.8 & 49.6 & \\
\hline Primary (4-8 yrs) & 25.3 & 28.6 & 23.7 & \\
\hline Secondary school or higher (>8 yrs) & 28.0 & 30.7 & 26.7 & \\
\hline Missing (N) & 103 & 36 & 67 & \\
\hline Working Status & & & & $<0.001$ \\
\hline Not working & 70.8 & 62.7 & 74.6 & \\
\hline Working & 29.2 & 37.3 & 25.4 & \\
\hline Missing (N) & 173 & 61 & 112 & \\
\hline Household Wealth Tertile & & & & 0.403 \\
\hline 1st (lowest wealth) & 34.1 & 32.5 & 34.9 & \\
\hline $2^{\text {nd }}$ & 21.3 & 20.9 & 21.5 & \\
\hline $3^{\text {rd }}$ & 44.6 & 46.6 & 43.6 & \\
\hline \multirow[t]{2}{*}{ Missing (N) } & 11 & 5 & 6 & \\
\hline & Mean & Mean & Mean & \\
\hline Systolic Blood Pressure & 133.80 & 133.6 & 133.9 & 0.789 \\
\hline Missing (N) & 44 & 23 & 21 & \\
\hline \multicolumn{5}{|l|}{ Systolic Blood Pressure by HIV Status } \\
\hline HIV Negative & 136.23 & 136.3 & 136.2 & 0.931 \\
\hline HIV Positive, Suppressed ${ }^{\mathrm{a}}$ & 126.24 & 125.6 & 126.5 & 0.780 \\
\hline HIV Positive, Unsuppressed ${ }^{\mathrm{b}}$ & 128.23 & 128.8 & 127.9 & 0.681 \\
\hline Missing (N) on both SBP and HIV & 35 & 21 & 14 & \\
\hline Died during 8-year follow-up & 28.5 & 36.9 & 24.5 & $<0.001$ \\
\hline$N$ & 2,909 & 1,107 & 1,802 & \\
\hline
\end{tabular}

Data come from Ha Nakekela HIV and Noncommunicable Disease Study and the Agincourt Health \& socio-Demographic Surveillance System. Proportions are survey weighted

${ }^{\text {a }}$ Suppressed if viral load $<400$ copies $/ \mathrm{mL}$

b Unsuppressed if viral load $\geq 400$ copies $/ \mathrm{mL}$ 
1,000 copies/mL [32]. We tested both systolic blood pressure and diastolic blood pressure. Since results were similar, we focus on systolic blood pressure but show diastolic blood pressure results in Additional File 1.

We structured the data into a person-year format, and used sex-stratified discrete-time models to characterize the risk of dying [33], adjusting for sample weighting and survey nonresponse [34]. The weights are a product of the inverse probability sample selection weights and inverse probability weights to adjust for nonresponse based on covariates from the 2009 census. Our discretetime model is similar to the Cox proportional hazards approach, but has a number of advantages, including: greater flexibility in modelling temporal relationships and interactions; and results in the form of predicted probabilities which can be interpreted independently. Respondents were followed until either July 2019, their death, or censoring (out-migration or loss-to-follow-up). To identify potential non-linear relationships between systolic blood pressure and mortality, we modelled mortality as a quadratic function of systolic blood pressure. Tests modelling systolic blood pressure as a restricted cubic spline showed similar results. We also adjusted for self-reported use of medications to lower blood pressure. We tested for interactions between age and HIV status, age and systolic blood pressure, and systolic blood pressure and HIV status using nested likelihood ratio tests.

\section{Results}

Table 1 presents descriptive results for the survey participants aged 40-plus. Over a quarter of men and women were living with HIV, over half of whom had unsuppressed viral loads in 2010-11. Figure 1 shows that the share of the population with a systolic blood pressure of $140 \mathrm{~mm} \mathrm{Hg}$ or higher significantly increased for men from $22 \%$ at ages $40-59$ to $48 \%$ at ages 75 -plus $(p<0.001)$ and for women from $25 \%$ to $51 \%(p<0.001)$. The proportion with a systolic blood pressure of $140 \mathrm{~mm} \mathrm{Hg}$ or more was generally higher for those not living with HIV compared to PLWH $(p<0.001$ for both men and

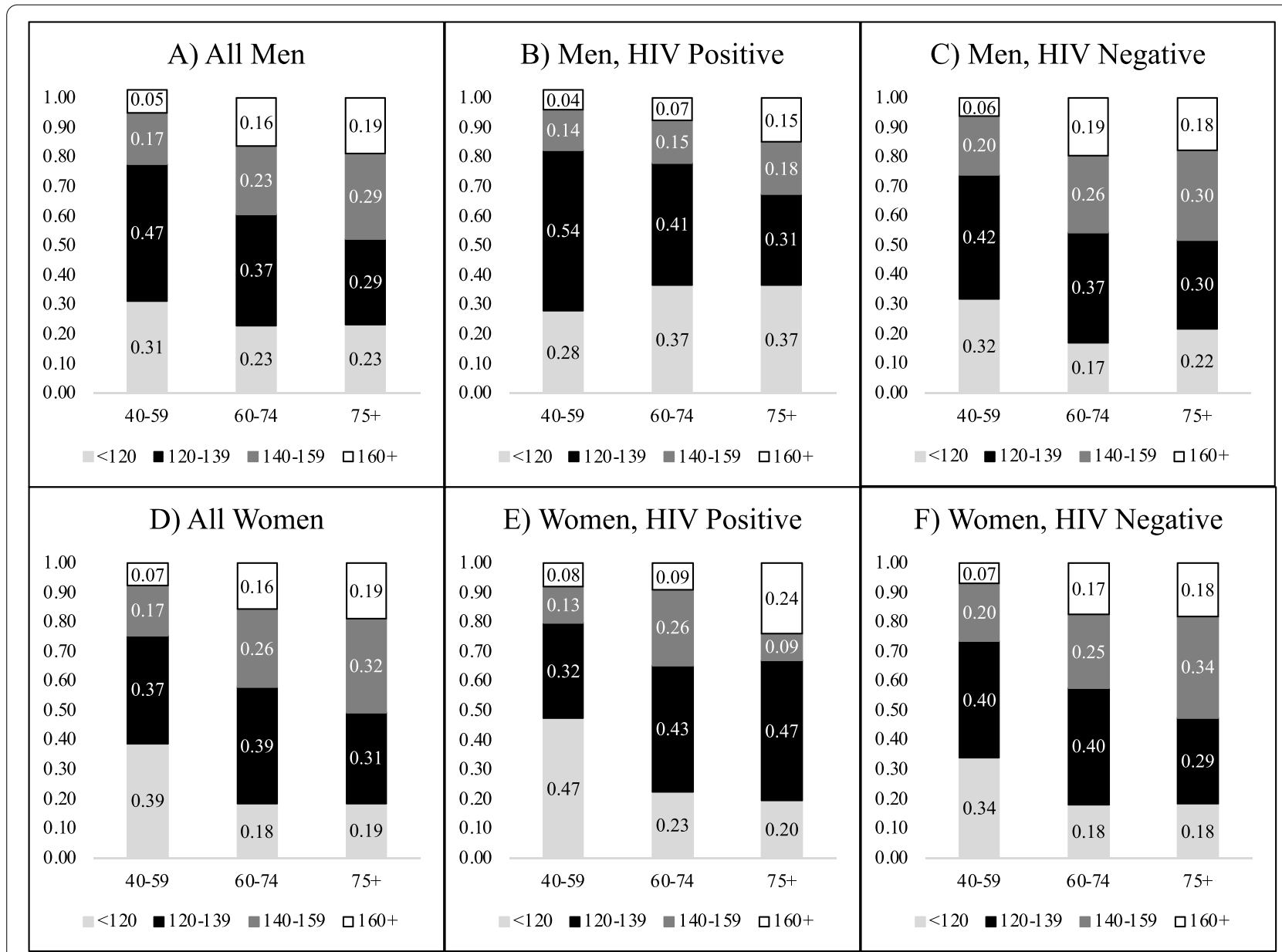

Fig. 1 Age-specific distributions of systolic blood pressure categories by sex and HIV status, Agincourt, South Africa, 2010-11. Proportions are survey weighted 
women), particularly at older ages. Mortality during the eight years of follow-up was high (477 deaths): survey weighted estimates are that $37 \%$ of men (mortality rate 987.53 per $100,000,95 \%$ CI: 986.26 to 988.79 ) and $25 \%$ of women (mortality rate 937.28 per 100,000, 95\% CI: 899.7 to 974.88 ) died.

Preliminary mortality models indicated that the only control variables significantly associated with mortality were marital status and education. We therefore omitted the other control variables to maximize sample size (supplemental models with all controls, no controls, and alternate viral load thresholds showed similar results to our final models, see Additional Files 2-3).

Table 2 presents the multivariate models. Results are similar for men and women. As expected, mortality increased with age and there was no significant additional mortality risk for PLWH with suppressed viral load. PLWH with unsuppressed viral load had higher odds of dying compared to those uninfected (men: adjusted odds ratio (aOR): $3.23,95 \%$ CI: 2.21 to 4.71 ; women: aOR: 2.05 , 95\% CI: 1.27 to 3.30). There was a weak, non-linear relationship between systolic blood pressure and mortality (shown in Fig. 2). Adding an interaction between HIV status and systolic blood pressure did not significantly improve model fit (men: $p=0.752$; women: $p=0.860$ ). Men diagnosed with hypertension and currently on hypertension medication had 1.52 times the odds of dying compared to all other men (95\% CI 1.05 to 2.21). Marriage and higher education were protective against mortality for men but showed no relationship for women.

The overall strength of the relationship between unsuppressed viral load and mortality was much greater than the relationship between systolic blood pressure and mortality. For instance, men with unsuppressed viral load had about a $5 \%$ higher probability of dying compared to uninfected men (95\% CI 0.029 to 0.074$)$. In contrast, the probability of dying was about $1 \%$ higher for men with a systolic blood pressure of $170 \mathrm{mmHg}$ compared to $140 \mathrm{mmHg}$ (95\% CI 0.002 to 0.018 ). In comparison, for women, there was a $1.6 \%$ higher probability of dying for women with unsuppressed viral load compared to uninfected women (95\% CI 0.002 to 0.030 ) and about $0.4 \%$ higher probability for women with a systolic blood pressure of $170 \mathrm{mmHg}$ compared to $140 \mathrm{mmHg}$ (95\% CI 0.0007 to 0.007 ).

\section{Discussion}

Our study highlights the effectiveness of HIV treatment in a rural South African population in which both men and women had high HIV and hypertension prevalence. Over an eight-year follow-up period, mortality of those with suppressed viral load was not significantly different

Table 2 Multivariable logistic regression of all-cause mortality on HIV status, systolic blood pressure, and baseline characteristics, Agincourt, South Africa, 2010-2019

\begin{tabular}{|c|c|c|c|c|c|c|}
\hline \multirow[b]{2}{*}{ Covariates } & \multicolumn{3}{|l|}{ Men $^{d}$} & \multicolumn{3}{|c|}{ Women $^{\mathrm{e}}$} \\
\hline & aOR & $95 \% \mathrm{Cl}$ & $P$-value & aOR & $95 \% \mathrm{Cl}$ & $P$-value \\
\hline Age & 1.05 & $(1.04,1.06)$ & $<0.001$ & 1.06 & $(1.04,1.07)$ & $<0.001$ \\
\hline \multicolumn{7}{|l|}{ HIV Status [ref: HIV negative] } \\
\hline HIV Positive Suppressed ${ }^{\mathrm{a}}$ & 1.62 & $(0.97,2.70)$ & 0.06 & 1.34 & $(0.76,2.34)$ & 0.31 \\
\hline HIV Positive, Unsuppressed ${ }^{\mathrm{b}}$ & 3.23 & $(2.21,4.71)$ & $<0.001$ & 2.05 & $(1.27,3.30)$ & 0.00 \\
\hline Systolic Blood Pressure & 0.93 & $(0.89,0.97)$ & $<0.001$ & 0.96 & $(0.92,1.01)$ & 0.10 \\
\hline Systolic Blood Pressure Squared ${ }^{c}$ & 1.00 & $(1.00,1.00)$ & $<0.001$ & 1.00 & $(1.00,1.00)$ & 0.05 \\
\hline Blood Pressure Medication & 1.52 & $(1.05,2.21)$ & 0.03 & 1.32 & $(0.97,1.80)$ & 0.08 \\
\hline \multicolumn{7}{|l|}{ Marital Status [ref: single] } \\
\hline Married/cohabiting & 0.48 & $(0.32,0.73)$ & $<0.001$ & 0.62 & $(0.35,1.09)$ & 0.10 \\
\hline Widowed/divorced & 0.74 & $(0.46,1.17)$ & 0.20 & 0.95 & $(0.57,1.59)$ & 0.84 \\
\hline \multicolumn{7}{|c|}{ Education Level [ref: none/very low ( $\leq 3$ years)] } \\
\hline Primary (4-8 years) & 0.69 & $(0.48,0.97)$ & 0.04 & 0.93 & $(0.61,1.40)$ & 0.72 \\
\hline Secondary school or higher ( $>8$ years) & 0.48 & $(0.28,0.83)$ & 0.01 & 0.85 & $(0.48,1.52)$ & 0.58 \\
\hline$N 2,692$ & 999 & & & 1,693 & & \\
\hline Person Years & 7,382 & & & 13,166 & & \\
\hline
\end{tabular}

${ }^{a}<400$ copies $/ \mathrm{mL}$

b $\geq 400$ copies $/ \mathrm{mL}$

${ }^{c}$ Coefficients and $95 \% \mathrm{Cl}$ are small numbers which appear as 1.000 due to rounding

${ }^{d}$ Joint test of systolic blood pressure and systolic blood pressure squared $\left(\chi_{2}^{2}=11.15 ; p=0.004\right)$

e Joint test of systolic blood pressure and systolic blood pressure squared $\left(\chi_{2}^{2}=10.19 ; p=0.006\right.$ ) 
from those who were not living with HIV. Unsuppressed viral load, however, was associated with significantly increased risk of mortality. There was also a weak, nonlinear concave relationship between systolic blood pressure and mortality risk.

Previous studies examining whether HIV infection and/or its treatment interact with blood pressure to increase mortality risk yielded mixed results [21-23]. In this study, we found no statistical interaction between HIV and systolic blood pressure in relation to mortality risk. However, our relatively small samples may have been underpowered to detect none but a large interaction.

A strength of the Ha Nakekela study is the use of a non-clinic, population-based sample including adults living with and without HIV. Our finding of elevated mortality for PLWH is important to situate in the local context. At the time of the baseline survey ART was just becoming widespread in the Agincourt HDSS study area $[35,36]$. Our finding of elevated mortality risks for men and women with unsuppressed viral load aligns with evidence from the Africa Health Research Institute's surveillance site (AHRI) in KwaZulu-Natal, which showed that mortality was increasingly concentrated in PLWH who had never sought care [37]. Continued mortality monitoring is needed to understand how this relationship may have changed over time, particularly as awareness and acceptance of HIV treatment became more widespread. Evidence from the ALPHA Network

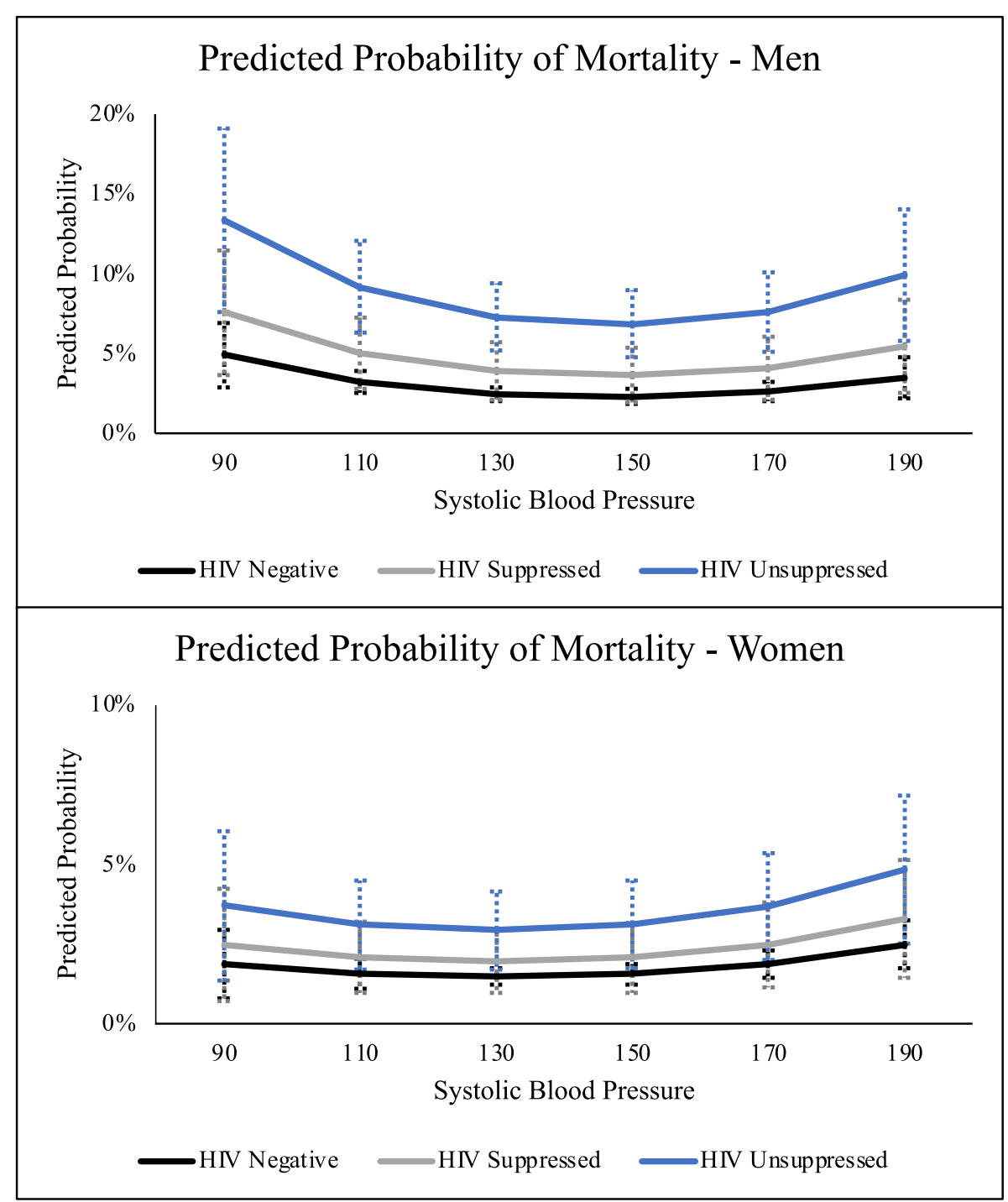

Fig. 2 Predicted probability of mortality by sex, HIV status and systolic blood pressure, Agincourt, South Africa, 2010-2018 
of demographic surveillance sites suggests that mortality among PLWH who are not on treatment is declining rapidly over time [38], which may be due in part to earlier diagnosis and treatment initiation so that untreated PLWH are increasingly people with early-stage disease.

Our finding of a non-linear relationship between systolic blood pressure and mortality aligns with results from a population-based cohort study at the national-level in South Africa, though they did not estimate separate models for men and women [39]. The authors suggested this weaker pattern compared to high-income countries may be due to competing mortality risks from $\mathrm{HIV}$ and TB predominately affecting ages 30-55 where systolic blood pressure is generally lower. Similarly, HIV/TB has also heavily impacted our study population, particularly in young adults [4]. Elevated mortality risks were associated with being on antihypertensive medication for men but not for women. It is possible that women are more likely to be engaged with the health care system [40], increasing their awareness and management of hypertension [10].

We found that the proportion of PLWH at baseline with a systolic blood pressure of $140 \mathrm{~mm} \mathrm{Hg}$ or more was lower compared to those who were not. This result accords with results from a longitudinal study in northwest South Africa that found that PLWH had a lower or similar blood pressure compared to those who were not living with HIV [41]. At the time of our study, under a third of men and just under half of women had suppressed viral loads. As ART has become more widespread, resulting survival gains, population ageing, and higher multimorbidity between HIV and cardiovascular risk factors may alter the patterns we observed [42, 43]. Further longitudinal follow-up with repeated measurements is needed in this population of older adults to assess the long-term cardiovascular effects of HIV and ART.

We acknowledge study strengths and limitations. We were able to include a long period of mortality follow-up. Our use of mortality data from population census updates also limits loss-to-follow-up, which has been shown to affect estimates from clinic-based follow-up studies [17]. Limitations include: that HIV status and systolic blood pressure were measured only at baseline; that despite high mortality, we lacked a sufficiently large sample size to investigate differential associations by cause of death; and that some respondents were excluded from the analysis due to missing data.

\section{Conclusions}

Our results indicate that HIV and elevated blood pressure are acting as separate, non-interacting epidemics affecting high proportions of the older adult population. Despite relatively high levels of treatment,
HIV continues to increase mortality for both men and women unless viral load is suppressed. Further, elevated blood pressure is increasing mortality for men and women. At present, the Agincourt HDSS is collecting data on COVID-19 in its surveillance site [44]. Future studies need to take into consideration the impacts of this third epidemic and its interactions with the advanced HIV and hypertension epidemics that continue to affect the lives of rural South Africans.

\section{Abbreviations}

aOR: Adjusted odds ratio; ART: Antiretroviral therapy; HDSS: Health and socioDemographic Surveillance System; NCD: Noncommunicable disease; PLWH: People living with HIV.

\section{Supplementary Information}

The online version contains supplementary material available at https://doi. org/10.1186/s12889-022-12791-z.

Additional file 1. Multivariable logistic regression of all-cause mortality on HIV status, diastolic blood pressure and baseline characteristics, Agincourt, South Africa, 2010-2019.

Additional file 2. Multivariable logistic regression of all-cause mortality for men on baseline characteristics: no covariates, all covariates, and alternate viral load threshold on all-cause mortality, Agincourt, South Africa, 2010-2019.

Additional file 3. Multivariable logistic regression of all-cause mortality for women on baseline characteristics: no covariates, all covariates, and alternate viral load threshold, Agincourt, South Africa, 2010-2019.

\section{Acknowledgements}

We thank all the respondents who participated in this study and the $\mathrm{Ha}$ Nakekela field team. We are also grateful to members of the HIV survey investigative team for their numerous contributions to the project. We also thank the people of the Agincourt sub-district for their long involvement with the MRC/ Wits Rural Public Health and Health Transitions Research Unit.

\section{Authors' contributions}

SM, JM, SC, ES, and NA designed the overall project. CK, FXG, NA, SC, and $\mathrm{JM}$ were involved in the data collection. $\mathrm{BH}$ and $\mathrm{JM}$ designed this study. $\mathrm{CK}$ curated the mortality data. AT conducted the analyses with supervision from $\mathrm{BH}$ and JM. All authors contributed to the writing and revision of the manuscript. All authors have read and approved the final manuscript.

\section{Funding}

We are grateful for funding support from: the US National Institute on Aging - R01 AG049634 HIV after 40 in rural South Africa: Aging in the Context of an HIV Epidemic (PI Sanyu Mojola); the National Institutes of Health-R24 AG032112-05 Partnership for Social Science AIDS Research in South Africa's Era of ART Rollout (PI Jane Menken); the University of Colorado, Innovative Seed Grant HIV after 40 in rural South Africa (PI Sanyu Mojola); the Eunice Kennedy Shriver National Institute of Child Health and Human Development-K01 HD057246 (PI Samuel Clark); and the William and Flora Hewlett Foundation 2009-4060 African Population Research and Training Program (PI Jane Menken). The MRC/Wits Rural Public Health and Health Transitions Research Unit and Agincourt Health and Socio-Demographic Surveillance System, a node of the South African Population Research Infrastructure Network (SAPRIN), is supported by the Department of Science and Innovation, the University of the Witwatersrand, and the Medical Research Council, South Africa, and previously the Wellcome Trust, UK (Grants 058893/Z/99/A; 069683/Z/02/Z; 085477/Z/08/Z; 085477/B/08/Z). This work has also benefited from research, administrative, and computing support from the Eunice Kennedy Shriver National Institute of Child Health 
and Human Development-funded University of Colorado Population Center (R24HD066613, P2C HD066613).

\section{Availability of data and materials}

The datasets used and/or analysed during the current study are available from the corresponding author on reasonable request.

\section{Declarations}

\section{Ethics approval and consent to participate}

This study received ethical approval from the authors institutions, the University of the Witwatersrand Human Research Ethics Committee (Medical M10458), and the Mpumalanga Provincial Research and Ethics Committee. All methods were carried out in accordance with relevant guidelines and regulations.

\section{Consent for publication}

Not applicable.

\section{Competing interests}

The authors declare that they have no competing interests.

\section{Author details}

${ }^{1}$ School of Demography, The Australian National University, Canberra, Australia. ${ }^{2}$ MRC/Wits Rural Public Health and Health Transitions Research Unit (Agincourt), Faculty of Health Sciences, School of Public Health, University of the Witwatersrand, Johannesburg, South Africa. ${ }^{3}$ Nuffield College, University of Oxford, Oxford, UK. ${ }^{4}$ Institute of Behavioral Science and Department of Sociology, University of Colorado Boulder, Boulder, USA. ${ }^{5}$ Leverhulme Centre for Demographic Science, Department of Sociology, University of Oxford, Oxford, UK. ${ }^{6}$ Department of Sociology, School of Public and International Affairs, and Office of Population Research, Princeton University, Princeton, USA. ${ }^{7}$ Department of Public Health, University of Missouri, Columbia, USA. ${ }^{8}$ Department of Sociology, The Ohio State University, Columbus, USA. ${ }^{9}$ Department of Sociology, American University, Washington, USA.

\section{Received: 25 October 2021 Accepted: 14 February 2022}

Published online: 24 February 2022

\section{References}

1. United Nations, Department of Economic and Social Affairs, Population Division. World Population Prospects 2019. 2019. http://www.popul ation.un.org/wpp/. Accessed 24 Sep 2020.

2. UNAIDS. Seizing the moment: Tackling entrenched inequalities to end epidemics. Global AIDS Update. 2020. http://www.unaids.org/en/resou rces/documents/2020/global-aids-report. Accessed 21 Aug 2020.

3. Bor J, Herbst AJ, Newell M-L, Bärnighausen T. Increases in adult life expectancy in rural South Africa: valuing the scale-up of HIV treatment. Science. 2013;339:961-5.

4. Kabudula CW, Houle B, Collinson MA, et al. Progression of the epidemiological transition in a rural South African setting: findings from population surveillance in Agincourt, 1993-2013. BMC Public Health. 2017;17:424.

5. Vollmer S, Harttgen K, Alfven T, Padayachy J, Ghys P, Bärnighausen T. The HIV epidemic in sub-Saharan Africa is aging: Evidence from the Demographic and Health Surveys in sub-Saharan Africa. AIDS Behav. 2016;21:101-13.

6. Rohr JK, Manne-Goehler J, Gómez-Olivé FX, et al. HIV treatment cascade for older adults in rural South Africa. Sex Transm Infect. 2020;96:271-6.

7. Mayosi BM, Flisher AJ, Lalloo UG, Sitas F, Tollman SM, Bradshaw D. The burden of non-communicable diseases in South Africa. Lancet. 2009:374:934-47.

8. Clark SJ, Gómez-Olivé FX, Houle B, et al. Cardiometabolic disease risk and HIV status in rural South Africa: establishing a baseline. BMC Public Health. 2015;15:372.

9. Lloyd-Sherlock P, Beard J, Minicuci N, Ebrahim S, Chatterji S. Hypertension among older adults in low- and middle-income countries: prevalence, awareness and control. International Journal of Epidemiology. 2014;43:116-28.

10. Gómez-Olivé FX, Ali SA, Made F, et al. Regional and Sex Differences in the Prevalence and Awareness of Hypertension: An H3Africa AWI-Gen Study Across 6 Sites in Sub-Saharan Africa. Glob Heart. 2017:12:81-90.

11. Jardim TV, Reiger S, Abrahams-Gessel S, et al. Hypertension management in a population of older adults in rural South Africa. Journal of Hypertension. 2017;35:1283-9.

12. Data Collection on Adverse Events of Anti-HIV Drugs (DAD) Study Group. Combination antiretroviral therapy and the risk of myocardial infarction. New England Journal of Medicine. 2003;349:1993-2003.

13. Nüesch $R$, Wang $Q$, Elzi $L$, et al. Risk of cardiovascular events and blood pressure control in hypertensive HIV-infected patients: Swiss HIV Cohort Study (SHCS). JAIDS Journal of Acquired Immune Deficiency Syndromes. 2013;62:396-404

14. Triant VA, Lee H, Hadigan C, Grinspoon SK. Increased acute myocardial infarction rates and cardiovascular risk factors among patients with human immunodeficiency virus disease. J Clin Endocrinol Metab. 2007;92:2506-12

15. Croxford S, Kitching A, Desai S, et al. Mortality and causes of death in people diagnosed with HIV in the era of highly active antiretroviral therapy compared with the general population: an analysis of a national observational cohort. The Lancet Public Health. 2017;2:e35-46.

16 Lohse N, Hansen A-BE, Pedersen G, et al. Survival of persons with and without HIV infection in Denmark, 1995-2005. Annals of Internal Medicine. 2007;146:87-95.

17. Geng EH, Odeny TA, Lyamuya RE, et al. Estimation of mortality among HIV-infected people on antiretroviral treatment in east Africa: a sampling based approach in an observational, multisite, cohort study. The Lancet HIV. 2015;2:e107-16.

18 Haachambwa L, Kandiwo N, Zulu PM, et al. Care continuum and postdischarge outcomes among HIV-infected adults admitted to the hospital in Zambia. Open Forum Infect Dis. 2019;6:ofz336.

19. Meintjes G, Kerkhoff AD, Burton R, et al. HIV-related medical admissions to a South African district hospital remain frequent despite effective antiretroviral therapy scale-up. Medicine. 2015;94:e2269.

20. Ousley J, Niyibizi AA, Wanjala S, et al. High proportions of patients with advanced HIV are antiretroviral therapy experienced: hospitalization outcomes from 2 sub-Saharan African sites. Clin Infect Dis. 2018;66:S126-31.

21. Batavia AS, Severe P, Lee MH, et al. Blood pressure and mortality in a prospective cohort of HIV-infected adults in Port-au-Prince. Haiti J Hypertens. 2018:36:1533-9.

22. Bloomfield GS, Hogan JW, Keter A, et al. Blood pressure level impacts risk of death among HIV seropositive adults in Kenya: a retrospective analysis of electronic health records. BMC Infect Dis. 2014;14:284.

23. Armah KA, Chang CC, Baker JV, et al. Prehypertension, hypertension, and the risk of acute myocardial infarction in HIV-infected and -uninfected veterans. Clin Infect Dis. 2014;58:121-9.

24. Kahn K, Collinson MA, Gomez-Olive FX, et al. Profile: Agincourt Health and Socio-demographic Surveillance System. Int J Epidemiol. 2012:41:988-1001.

25. Gómez-Olivé FX, Angotti N, Houle B, et al. Prevalence of HIV among those 15 and older in rural South Africa. AIDS Care. 2013:25:1122-8.

26. Houle B, Mojola SA, Angotti N, et al. Sexual behavior and HIV risk across the life course in rural South Africa: trends and comparisons. AIDS Care. 2018;30:1435-43.

27. Altunkan S, Öztas K, Altunkan E. Validation of the Omron 637IT wrist blood pressure measuring device with a position sensor according to the International Protocol in adults and obese adults. Blood Pressure Monitoring. 2006:11:79-85.

28. Omboni S, Riva I, Giglio A, Caldara G, Groppelli A, Parati G. Validation of the Omron M5-I, R5-I and HEM-907 automated blood pressure monitors in elderly individuals according to the International Protocol of the European Society of Hypertension. Blood Pressure Monitoring. 2007; 12:233-42.

29. Saladini F, Benetti E, Palatini P. Accuracy of the visomat handy wrist blood pressure measuring device according to the International Protocol. Blood Press Monit. 2010;15:281-4

30. Kabudula C, Houle B, Collinson MA, Kahn K, Tollman S, Clark S. Assessing changes in household socioeconomic status in rural South Africa, 
2001-2013: a distributional analysis using household asset indicators. Social Indicators Research. 2016;133:1047-73.

31. Dawood H, Hassan-Moosa R, Zuma NY, Naidoo K. Mortality and treatment response amongst HIV-infected patients 50 years and older accessing antiretroviral services in South Africa. BMC Infect Dis. 2018;18:168.

32 Republic of South Africa National Department of Health. 2019 ART Clinical Guidelines for the Management of HIV in Adults Pregnancy Adolescents, Children, Infants and Neonates. 2019.

33. Allison PD. Discrete-Time Methods for the Analysis of Event Histories. Sociological Methodology. 1982;13:61-98.

34. Schatz E, Houle B, Mojola SA, Angotti N, Williams J. How to 'live a good life': aging and HIV testing in rural South Africa. Journal of Aging and Health. 2019;31:709-32.

35. Houle B, Clark SJ, Gómez-Olivé FX, Kahn K, Tollman SM. The unfolding counter-transition in rural South Africa: mortality and cause of death, 1994-2009. PLoS One. 2014;9:e100420.

36. Mee P, Collinson MA, Madhavan S, et al. Evidence for localised HIV related micro-epidemics associated with the decentralised provision of antiretroviral treatment in rural South Africa: a spatio-temporal analysis of changing mortality patterns (2007-2010). J Glob Health. 2014:4:010403.

37. Bor J, Rosen S, Chimbindi N, et al. Mass HIV treatment and sex disparities in life expectancy: demographic surveillance in rural South Africa. PLoS medicine. 2015;12:e1001905.

38. Reniers G, Slaymaker E, Nakiyingi-Miiro J, et al. Mortality trends in the era of antiretroviral therapy: evidence from the Network for Analysing Longitudinal Population based HIV/AIDS data on Africa (ALPHA). AIDS. 2014;28(Suppl 4):S533-42.

39. Diallo AO, Ali MK, Geldsetzer P, et al. Systolic blood pressure and 6-year mortality in South Africa: a country-wide, population-based cohort study. The Lancet Healthy Longevity. 2021;2:e78-86.

40. Gómez-Olivé FX, Thorogood M, Clark B, Kahn K, Tollman S. Self-reported health and health care use in an ageing population in the Agincourt subdistrict of rural South Africa. Global Health Action. 2013;6:19305.

41. Schutte AE, Schutte R, Huisman HW, et al. Are behavioural risk factors to be blamed for the conversion from optimal blood pressure to hypertensive status in Black South Africans? A 5-year prospective study. International Journal of Epidemiology. 2012;41:1114-23.

42. Chang AY, Gómez-Olivé FX, Manne-Goehler J, et al. Multimorbidity and care for hypertension, diabetes and HIV among older adults in rural South Africa. Bull World Health Organ. 2019;97:10-23.

43. Sudharsanan N, Geldsetzer P. Impact of Coming Demographic Changes on the Number of Adults in Need of Care for Hypertension in Brazil, China, India, Indonesia, Mexico, and South Africa. Hypertension. 2019;73:770-6.

44. Harling G, Gómez-Olivé FX, Tlouyamma J, et al. Protective Behaviors and Secondary Harms Resulting From Nonpharmaceutical Interventions During the COVID-19 Epidemic in South Africa: Multisite, Prospective Longitudinal Study. JMIR Public Health and Surveillance. 2021;7:e26073.

\section{Publisher's Note}

Springer Nature remains neutral with regard to jurisdictional claims in published maps and institutional affiliations.

Ready to submit your research? Choose BMC and benefit from:

- fast, convenient online submission

- thorough peer review by experienced researchers in your field

- rapid publication on acceptance

- support for research data, including large and complex data types

- gold Open Access which fosters wider collaboration and increased citations

- maximum visibility for your research: over $100 \mathrm{M}$ website views per year

At BMC, research is always in progress.

Learn more biomedcentral.com/submissions 